\title{
DYNAMICAL AND CURVATURE TRAJECTORIES IN SPACE
}

\author{
BY \\ JOHN DECICCO
}

1. Dynamical trajectories. Kasner in his Princeton Colloquium Lectures ${ }^{(1)}$ studied the geometry of the dynamical trajectories in the plane and in space. In the plane, a completely characteristic set of five properties were obtained; and in space, a completely characteristic set of four properties were given.

Consider the motion of a particle of unit mass in space under the action of any positional field of force. The general equations of motion are

$$
d^{2} x / d t^{2}=\phi(x, y, z), \quad d^{2} y / d t^{2}=\psi(x, y, z), \quad d^{2} z / d t^{2}=\chi(x, y, z),
$$

where $(\phi, \psi, \chi)$ are the rectangular components of the force acting at any point $(x, y, z)$.

The total number of trajectories for all initial conditions is $\infty^{5}$. By eliminating the time $t$ from the equations (1), it is found that the dynamical trajectories of a positional field of force in space are given by the $\infty^{5}$ integral curves which are the complete solutions of the Monge equation of second $\operatorname{order}\left({ }^{2}\right)$

$$
z^{\prime \prime}=\frac{x-z^{\prime} \phi}{\psi-y^{\prime} \phi} y^{\prime \prime}
$$

solved together with the Monge equation of third order

$$
y^{\prime \prime \prime}=\frac{\left[\left(\psi_{x}+y^{\prime} \psi_{y}+z^{\prime} \psi_{z}\right)-y^{\prime}\left(\phi_{x}+y^{\prime} \phi_{y}+z^{\prime} \phi_{z}\right)\right]}{\psi-y^{\prime} \phi} y^{\prime \prime}-\frac{3 \phi}{\psi-y^{\prime} \phi} y^{\prime \prime 2} .
$$

2. The curvature trajectories. We begin with any system of $\infty^{4}$ curves (not all straight lines). Any such system of $\infty^{4}$ curves may be given as the complete solutions of the simultaneous pair of Monge equations of second order

$$
y^{\prime \prime}=e^{F}, \quad z^{\prime \prime}=e^{F} K
$$

Presented to the Society, November 26, 1943; received by the editors July 1, 1944.

(1) Kasner, Differential-geometric aspects of dynamics, Amer. Math. Soc. Colloquium Publications, vol. 3, 1913, 1934. Also see a series of papers in Trans. Amer. Math. Soc. vols. 7-11 (1906-1910).

(2) Throughout this paper, primes denote total differentiation with respect to $x$, whereas the subscripts $x, y, z, y^{\prime}, z^{\prime}$, denote partial differentiation with respect to each of the five variables. 
where $F$ and $K$ are functions of $x, y, z, y^{\prime}$ and $z^{\prime}$. (The differential equations of $\infty^{4}$ curves, not all straight lines, may always be written in these forms by appropriately interchanging $y$ and $z$.) A curvature trajectory of this family is a curve which is drawn so that at each point it has the same osculating plane as, and also $c$ times the curvature of, the member of the family to which it is tangent at that point, $c$ remaining constant along the trajectory. For a given value of $c$ there will be a set of $\infty^{4}$ curvature trajectories, one in each direction through each point. By varying $c$, there result $\infty^{1}$ such sets. Hence a given quadruply-infinite family (4) generates a quintuply-infinite family of curvature trajectories, which are defined by the pair of simultaneous differential equations

$$
z^{\prime \prime}=K y^{\prime \prime}, \quad y^{\prime \prime \prime}=\left(F_{x}+y^{\prime} F_{y}+z^{\prime} F_{z}\right) y^{\prime \prime}+\left(F_{y^{\prime}}+K F_{z^{\prime}}\right) y^{\prime \prime 2} .
$$

Kasner has discussed the innate projective character of dynamical families in the Princeton Colloquium. The concept of curvature trajectories is also projective. This is an immediate consequence of Mehmke's theorem, which states that if two curves are tangent at a point, the ratio of the curvatures is a projective invariant. This theorem demonstrates that the entire process of construction of curvature trajectories has projective meaning.

3. The three-dimensional type $(G)$. Now it is observed that the $\infty^{5} \mathrm{dy}$ namical trajectories defined by (2) and (3) and the curvature trajectories defined by (5) are all special cases of systems of $\infty^{5}$ curves of the three-dimensional type $(G)$ given by differential equations of the forms

$$
\begin{aligned}
z^{\prime \prime} & =K\left(x, y, z, y^{\prime}, z^{\prime}\right) y^{\prime \prime}, \\
y^{\prime \prime \prime} & =G\left(x, y, z, y^{\prime}, z^{\prime}\right) y^{\prime \prime}+H\left(x, y, z, y^{\prime}, z^{\prime}\right) y^{\prime \prime 2} .
\end{aligned}
$$

Kasner has shown that such systems of $\infty^{5}$ curves of the three-dimensional type $(G)$ are characterized geometrically by the following two properties $\left(^{3}\right)$.

Property I. The $\infty^{1}$ curves which pass through a given lineal element $E$ all have the same osculating plane.

PROPERTy II. If the osculating spheres are constructed at the lineal element $E$ to the $\infty^{1}$ curves passing through $E$, the centers describe a straight line.

4. The statement of the problem. This common resemblance between dynamical and curvature trajectories suggests the problem of determining all quintuply-infinite systems of curves in space which are at once dynamical and curvature trajectories.

Kasner has completely solved this problem in the plane. The appropriate

(3) See Kasner and DeCicco, Generalized dynamical trajectories in space, Bull. Amer. Math. Soc. abstract 49-3-120; Duke Math. J. vol. 10 (1943) pp. 733-742. Also see DeCicco, Extension of certain dynamical theorems of Halphen and Kasner, Bull. Amer. Math. Soc. vol. 49 (1943). 
families in the plane are exactly the trajectories of all central or parallel fields of force $\left({ }^{4}\right)$.

It is our purpose to consider the above problem in space. We shall prove the following result.

Fundamental Theorem. The systems of $\infty^{5}$ curves which are simultaneously dynamical and curvature trajectories are the dynamical trajectories of the following three distinct types of fields of force:

(I). Those whose lines of force all lie in a pencil of planes.

(II). Those whose lines of force are orthogonal to a family of $\infty^{2}$ circular helices, all of which possess the same axis and the same period.

(III). Those of the central or parallel type.

Thus the answer in space contains more types than in the plane. We note that each of these types is projectively invariant.

5. The beginning of the proof of our Fundamental Theorem. We proceed with the proof. First let $\alpha$ and $\beta$ be functions of $(x, y, z)$ defined by

$$
\alpha=\psi / \phi, \quad \beta=\chi / \phi .
$$

Thus $(1, \alpha, \beta)$ are the direction numbers of the line of force of the force acting at the point $(x, y, z)$. Therefore the differential equations (2) and (3) defining the $\infty^{5}$ dynamical trajectories may be written as

$$
\begin{aligned}
z^{\prime \prime} & =\frac{\beta-z^{\prime}}{\alpha-y^{\prime}} y^{\prime \prime}, \\
y^{\prime \prime \prime} & =\left[\frac{\alpha_{x}+y^{\prime} \alpha_{y}+z^{\prime} \alpha_{z}}{\alpha-y^{\prime}}+\frac{\phi_{x}+y^{\prime} \phi_{y}+z^{\prime} \phi_{z}}{\phi}\right] y^{\prime \prime}-\frac{3}{\alpha-y^{\prime}} y^{\prime \prime 2} .
\end{aligned}
$$

For the trajectories of (5) and (7) to be at once dynamical and also curvature, it is seen that we must have

$$
\begin{gathered}
K=\frac{\beta-z^{\prime}}{\alpha-y^{\prime}}, \quad F_{y^{\prime}}+K F_{z^{\prime}}=-\frac{3}{\alpha-y^{\prime}}, \\
F_{x}+y^{\prime} F_{y}+z^{\prime} F_{z}=\frac{\alpha_{x}+y^{\prime} \alpha_{y}+z^{\prime} \alpha_{z}}{\alpha-y^{\prime}}+\frac{\phi_{x}+y^{\prime} \phi_{y}+z^{\prime} \phi_{z}}{\phi} .
\end{gathered}
$$

By the first two of the preceding equations, it is seen that the most general form of $F$ is

$$
F=3 \log \left(\alpha-y^{\prime}\right)+\log \phi+2 \log G(x, y, z, K),
$$

where $G \neq 0$.

(4) Kasner, Dynamical trajectories and curvature trajectories, Bull. Amer. Math. Soc. vol. 44 (1934) pp. 449-455. Also Comenetz, Amer. J. Math. (1935). DeCicco, Dynamical trajectories of the curvature type, Proc. Nat. Acad. Sci. U. S. A. vol. 29 (1943) pp. 268-270. 
Substituting this into the last of equations (8), we find (10) $\frac{\alpha_{x}+y^{\prime} \alpha_{y}+z^{\prime} \alpha_{z}}{\alpha-y^{\prime}}+\frac{1}{G}\left[\left(G_{x}+y^{\prime} G_{y}+z^{\prime} G_{z}\right)+G_{K}\left(K_{x}+y^{\prime} K_{y}+z^{\prime} K_{z}\right)\right]=0$

Next eliminating the partial derivatives of $K$ from the above equation by means of the first of equations (8), we find that the preceding equation becomes

$$
\begin{aligned}
\left(\alpha-y^{\prime}\right)\left(G_{x}+\right. & \left.y^{\prime} G_{y}+z^{\prime} G_{z}\right)+G\left(\alpha_{x}+y^{\prime} \alpha_{y}+z^{\prime} \alpha_{z}\right) \\
& +G_{K}\left[\left(\beta_{x}+y^{\prime} \beta_{y}+z^{\prime} \beta_{z}\right)-K\left(\alpha_{x}+y^{\prime} \alpha_{y}+z^{\prime} \alpha_{z}\right)\right]=0 .
\end{aligned}
$$

Now upon eliminating $z^{\prime}$ from this equation and the first of equations (8), we find

$$
\begin{aligned}
\left(\alpha-y^{\prime}\right)\left[\left\{G_{x}+(\beta\right.\right. & \left.\left.-K \alpha) G_{z}\right\}+y^{\prime}\left\{G_{y}+K G_{z}\right\}\right] \\
& +G\left[\left\{\alpha_{x}+(\beta-K \alpha) \alpha_{z}\right\}+y^{\prime}\left\{\alpha_{y}+K \alpha_{z}\right\}\right] \\
& +G_{K}\left[\left\{\left(\beta_{x}-K \alpha_{x}\right)+(\beta-K \alpha)\left(\beta_{z}-K \alpha_{z}\right)\right\}\right. \\
& \left.+y^{\prime}\left\{\left(\beta_{y}-K \alpha_{y}\right)+K\left(\beta_{z}-K \alpha_{z}\right)\right\}\right]=0 .
\end{aligned}
$$

This is an identity in $y^{\prime}$. We obtain the equations

$$
\begin{aligned}
& G_{y}+K G_{z}=0, \\
& G\left(\alpha_{y}+K \alpha_{z}\right)+G_{K}\left[\left(\beta_{y}+K \beta_{z}\right)-K\left(\alpha_{y}+K \alpha_{z}\right)\right]=G_{x}+(\beta-K \alpha) G_{z}, \\
& G\left(\alpha_{x}+\alpha \alpha_{y}+\beta \alpha_{z}\right)+G_{K}\left[\left(\beta_{x}+\alpha \beta_{y}+\beta \beta_{z}\right)-K\left(\alpha_{x}+\alpha \alpha_{y}+\beta \alpha_{z}\right)\right]=0 .
\end{aligned}
$$

These are the equations that we must solve for $G(x, y, z, K), \alpha(x, y, z)$ and $\beta(x, y, z)$.

Now we shall change the independent variable $z$ to the new independent variable $u$ by the substitution

$$
z=u+K y .
$$

Therefore $G, \alpha, \beta$ all become functions of $(x, y, u, K)$ satisfying the simultaneous partial differential equations

$$
\begin{aligned}
& G_{y}=0, \quad \alpha_{K}=y \alpha_{u}, \quad \beta_{K}=y \beta_{u}, \\
& G \alpha_{y}+G_{K}\left(\beta_{y}-K \alpha_{y}\right)=G_{x}+G_{u}\left[(\beta-K \alpha)+y\left(\beta_{y}-K \alpha_{y}\right)\right], \\
& {\left[\alpha_{x}+\alpha \alpha_{y}+(\beta-K \alpha) \alpha_{u}\right]\left[G-K\left(G_{K}-y G_{u}\right)\right]} \\
& \quad+\left[\beta_{x}+\alpha \beta_{y}+(\beta-K \alpha) \beta_{u}\right]\left[G_{K}-y G_{u}\right]=0 .
\end{aligned}
$$

The second and third equations are the conditions that $\alpha$ and $\beta$ be independent of $K$ when the variable $u$ is changed back to $z$ by means of (14).

It must be emphasized that by means of the transformation (14), the independent variables $(x, y, z, K)$ have been changed to a new set of independent variables $(x, y, u, K)$, and therefore the partial derivatives appearing in equa- 
tions (15) are entirely different from the similar ones appearing in equations (13).

Thus we have $G=G(x, u, K)$ only. Integrating the fourth of the preceding equations with respect to $y$ and rearranging the terms, we find

$$
\left(G_{K}-y G_{x}\right) \beta=\alpha\left[-G+K\left(G_{K}-y G_{u}\right)\right]+\left[y G_{x}+H(x, u, K)\right] .
$$

6. Case 1. $G_{K}-y G_{u}=0$. We discuss the case where the coefficient of $\beta$ in the preceding equation is identically zero. Thence $G=G(x)$ only and we have from the above equation that

$$
\alpha=(1 / G)\left(y G_{x}+H\right) .
$$

Substituting this into the condition $\alpha_{K}=y \alpha_{u}$, we discover that $H$ is a function of $x$ only; so that in the above $G=G(x)$ and $H=H(x)$.

Finally substituting the above into the last of equations (15), we find that $y G_{x x}+H_{x}=0$. Hence $\alpha$ is of the form

$$
\alpha=\left(a_{0} y+c_{0}\right) /\left(a_{0} x+b_{0}\right),
$$

where $\left(a_{0}, b_{0}, c_{0}\right)$ are constants.

If $a_{0}=0$, the lines of force lie in a pencil of parallel planes, all of which are parallel to the $z$-axis. If $a_{0} \neq 0$, the lines of force lie in a pencil of planes with axis $x=-b_{0} / a_{0}, y=-c_{0} / a_{0}$, which is parallel to the $z$-axis. Thus Case 1 belongs to the Type (I) of our Fundamental Theorem.

7. The case where $G_{K}-y G_{u} \neq 0$. Henceforth we may assume that $G$ is not a function of $x$ alone. Then we can solve (16) for $\beta$ obtaining

$$
\beta=\alpha\left[\frac{-G}{G_{K}-y G_{u}}+K\right]+\frac{y G_{z}+H}{G_{K}-y G_{u}} .
$$

Substituting this into the equations $\beta_{K}=y \beta_{u}$ and $\alpha_{K}=y \alpha_{u}$, we find

$$
\begin{aligned}
G( & \left.y^{2} G_{u u}-2 y G_{u K}+G_{K K}\right) \alpha \\
= & y^{2}\left(G_{x} G_{u u}-G_{u} G_{x u}\right)+y^{2}\left(G_{K} G_{x u}+G_{u} G_{x K}-2 G_{x} G_{u K}-G_{u} H_{u}+H G_{u u}\right) \\
& +y\left(G_{z} G_{K K}-G_{K} G_{x K}+G_{K} H_{u}+G_{u} H_{K}-2 H G_{u K}\right)+\left(H G_{K K}-G_{K} H_{K}\right) .
\end{aligned}
$$

8. Case 2. $y^{2} G_{u u}-2 y G_{u K}+G_{K K}=0$. In this case, the above equation must then be an identity in $y$ so that

$$
\begin{gathered}
G_{u u}=G_{u K}=G_{K K}=G_{u} G_{x u}=G_{K} H_{K}=0, \\
G_{u}\left(G_{x K}-H_{u}\right)=0, \quad G_{K}\left(G_{x K}-H_{u}\right)-G_{u} H_{K}=0 .
\end{gathered}
$$

If $G_{u} \neq 0$, these equations become equivalent to the equations

$$
\begin{gathered}
G_{u u}=G_{u K}=G_{K K}=G_{x u}=H_{K}=0, \\
G_{x K}-H_{u}=0 .
\end{gathered}
$$


On the other hand if $G_{u}=0$, then $G_{K} \neq 0$. For otherwise $G$ would contain $x$ only. Hence from (21), we again obtain the preceding equations. Thus in all cases we have the above conditions.

The equations (22) show that $G$ and $H$ are given by expressions of the forms

$$
\begin{aligned}
& G=e_{0} u+a K+c, \\
& B=a_{x} u+\lambda,
\end{aligned}
$$

where $e_{0}$ is a constant and $(a, c, \lambda)$ are functions of $x$ only. The equation (19) then becomes

$$
\beta=-\alpha\left[\frac{e_{0}(u+K y)+c}{a-e_{0} y}\right]+\left[\frac{y\left(a_{x} K+c_{x}\right)+\left(a_{x} u+\lambda\right)}{a-e_{0} y}\right] .
$$

Finally substituting the above into the last of equations (15), we find that the terms containing $\alpha$ disappear, and we find the identity

$$
y\left(a_{x x} K+c_{x x}\right)+\left(a_{x x} u+\lambda_{x}\right)=0 .
$$

From this we find $a_{x x}=c_{x x}=\lambda_{x}=0$. Therefore $a=a_{0} x+b_{0}, c=-c_{0} x-d_{0}, \lambda=\lambda_{0}$, where $\left(a_{0}, b_{0}, c_{0}, d_{0}, \lambda_{0}\right)$ are constants. Hence $\alpha$ and $\beta$ satisfy the single relation

$$
\alpha\left(e_{0} z-c_{0} x-d_{0}\right)-\beta\left(e_{0} y-a_{0} x-b_{0}\right)+\left(c_{0} y-a_{0} z-\lambda_{0}\right)=0 .
$$

Let us first of all consider the case where $e_{0}=0$ and $a_{0}=0$. Then since $G$ is not a function of $x$ only, we see by (23) that $b_{0} \neq 0$. In the above equation, we may then divide by $b_{0}$ and so take $b_{0}=1$. The above then becomes

$$
\beta=\alpha\left(c_{0} x+d_{0}\right)-\left(c_{0} y-\lambda_{0}\right) .
$$

If $c_{0}=0$, the lines of force lie in the family of parallel planes $z=\lambda_{0} x+d_{0} y$ + const. These fields of force then come under the Type (I) of our Fundamental Theorem.

Next let $c_{0} \neq 0$. Apply the translation

$$
X=x+d_{0} / c_{0}, \quad Y=y-\lambda_{0} / c_{0}, \quad Z=z
$$

to the equation (27). Thence we have the relation $B=c_{0}(A X-Y)$, where $(1, A, B)$ are the direction numbers of the line of force acting at the point $(X, Y, Z)$. The lines of force are then orthogonal to the system of $\infty^{2}$ curves

$$
d X / Y=d Y /-X=c_{0} d Z / 1 \text {. }
$$

Integrating the preceding equations, we find

$$
X=r_{0} \cos c_{0}\left(Z-Z_{0}\right), \quad Y=r_{0} \sin c_{0}\left(Z-Z_{0}\right),
$$

where $r_{0}$ and $Z_{0}$ are the two constants of integration. These are then a system of $\infty^{2}$ helices with the same axis whose equations are in the old coordinate system $x=-d_{0} / c_{0}, y=\lambda_{0} / c_{0}$ and with the same period $2 \pi / c_{0}$. Therefore the 
fields of force for the case $c_{0} \neq 0$ come under the Type (II) of our Fundamental Theorem.

Next suppose that $e_{0}=0$ but $a_{0} \neq 0$. We can divide (26) by $a_{0}$ and therefore $a_{0}$ can be taken to be unity. Hence (26) may be written in the form

$$
\beta=\alpha\left(\frac{\delta_{0}}{x-x_{0}}+c_{0}\right)+\frac{z-c_{0} y+\lambda_{0}}{x-x_{0}} .
$$

If $\delta_{0}=0$, the lines of force lie in the pencil of planes with axis $x=x_{0}$, $z=c_{0} y-\lambda_{0}$. These fields of force come under the Type (I) of our Fundamental Theorem.

Next consider the case where $\delta_{0} \neq 0$. Let us apply the rigid motion

$$
X=x-x_{0}+\frac{\delta_{0} c_{0}}{1+c_{0}^{2}}, \quad Y=\frac{y+c_{0} z}{\left(1+c_{0}^{2}\right)^{1 / 2}}, \quad Z=\frac{z-c_{0} y+\lambda_{0}}{\left(1+c_{0}^{2}\right)^{1 / 2}}
$$

to our equation (31). We find that it can be written in the form

$$
\left(1+c_{0}^{2}\right) X B=\delta_{0} A+\left(1+c_{0}^{2}\right) Z,
$$

where $(1, A, B)$ are the new direction numbers of the line of force acting at the point $(X, Y, Z)$. The lines of force must therefore be orthogonal to the $\infty^{2}$ curves defined by

$$
d X / Z=\left(1+c_{0}^{2}\right) d Y / \delta_{0}=d Z /-X .
$$

Integrating, we find the $\infty^{2}$ helices

$$
X=r_{0} \cos \frac{\left(1+c_{0}^{2}\right)}{\delta_{0}}\left(Y-Y_{0}\right), \quad Z=r_{0} \sin \frac{\left(1+c_{0}^{2}\right)}{\delta_{0}}\left(Y-Y_{0}\right),
$$

where $r_{0}$ and $Y_{0}$ are the constants of integration. These are a system of $\infty^{2}$ helices with the same axis $x=x_{0}-\delta_{0} c_{0} /\left(1+c_{0}^{2}\right), z=c_{0} y-\lambda_{0}$, and the same period $2 \pi \delta_{0} /\left(1+c_{0}^{2}\right)$. Thus these fields of force come under the Type (II).

Finally let us consider the case where $e_{0} \neq 0$. Upon dividing (26) by $e_{0}$, we may then take $e_{0}$ to be unity. It is found that (26) may be written in the form

$$
\left(\alpha-a_{0}\right)\left(z-c_{0} x-d_{0}\right)-\left(\beta-c_{0}\right)\left(y-a_{0} x-b_{0}\right)=E_{0} .
$$

If $E_{0}=0$, this means that the lines of force all lie in a pencil of planes with axis $y=a_{0} x+b_{0}, z=c_{0} x+d_{0}$. These fields of force come under the general Type (I).

We next discuss the case where $E_{0} \neq 0$. Let $R_{1}$ be the rigid motion

$$
R_{1}: X_{1}=\frac{x+a_{0}\left(y-b_{0}\right)}{\left(1+a_{0}^{2}\right)^{1 / 2}}, \quad Y_{1}=\frac{a_{0} x-\left(y-b_{0}\right)}{\left(1+a_{0}^{2}\right)^{1 / 2}}, \quad Z_{1}=z-d_{0} ;
$$


and let $R_{2}$ be the second rigid motion

$$
\begin{aligned}
& X=\frac{\left(1+a_{0}^{2}\right)^{1 / 2} X_{1}+c_{0} Z_{1}}{\left(1+a_{0}^{2}+c_{0}^{2}\right)^{1 / 2}}, \quad Y=Y_{1}+\frac{c_{0} E_{0}}{\left(1+a_{0}^{2}\right)^{1 / 2}\left(1+a_{0}^{2}+c_{0}^{2}\right)}, \\
& Z=\frac{-c_{0} X_{1}+\left(1+a_{0}^{2}\right)^{1 / 2} Z_{1}}{\left(1+a_{0}^{2}+c_{0}^{2}\right)^{1 / 2}}+\frac{a_{0} E_{0}}{\left[\left(1+a_{0}^{2}\right)\left(1+a_{0}^{2}+c_{0}^{2}\right)\right]^{1 / 2}} .
\end{aligned}
$$

Upon applying the product $R=R_{2} R_{1}$ of these two rigid motions to (36), we find that it becomes

$$
Y B=Z A+E_{0} /\left(1+a_{0}^{2}+c_{0}^{2}\right),
$$

where $(1, A, B)$ are the new direction numbers of the line of force acting at the point $(X, Y, Z)$. Therefore the lines of force are orthogonal to the system of $\infty^{2}$ curves given by

$$
\left(1+a_{0}^{2}+c_{0}^{2}\right) d X / E_{0}=d Y / Z=d Z /-Y .
$$

The integration of these yield

$$
Y=r_{0} \cos \frac{\left(1+a_{0}^{2}+c_{0}^{2}\right)}{E_{0}}\left(X-X_{0}\right), Z=r_{0} \sin \frac{\left(1+a_{0}^{2}+c_{0}^{2}\right)}{E_{0}}\left(X-X_{0}\right),
$$

where $r_{0}$ and $X_{0}$ are the constants of integration. This is a family of $\infty^{2}$ helices with the same axis whose equations are in the original coordinate system

$$
\begin{aligned}
& y=a_{0} x+b_{0}+c_{0} E_{0} /\left(1+a_{0}^{2}+c_{0}^{2}\right), \\
& z=c_{0} x+d_{0}-a_{0} E_{0} /\left(1+a_{0}^{2}+c_{0}^{2}\right),
\end{aligned}
$$

and with the same period $2 \pi E_{0} /\left(1+a_{0}^{2}+c_{0}^{2}\right)$. Thus these fields of force come under the general Type (II) of our Fundamental Theorem.

9. The case where $y^{2} G_{u u}-2 y G_{u K}+G_{K K} \neq 0$. Substitute the value of $\beta$ as given by (19) into the last of equations (15). We obtain the quadratic equation in $\alpha$

$$
\begin{aligned}
G^{2}\left(y G_{u u}-\right. & \left.G_{u K}\right) \alpha^{2}+G\left[2\left(y G_{x}+H\right)\left(G_{u K}-y G_{u u}\right)\right. \\
& \left.+\left(G_{K}-y G_{u}\right)\left\{\left(G_{x K}-H_{u}\right)-2 y G_{x u}\right\}\right] \alpha \\
& +\left(G_{K}-y G_{u}\right)^{2}\left(y G_{x x}+H_{x}\right)-\left(y G_{x}+H\right)^{2}\left(G_{u K}-y G_{u u}\right) \\
& -\left(G_{K}-y G_{u}\right)\left(y G_{x}+H\right)\left[\left(G_{x K}-H_{u}\right)-2 y G_{x u}\right]=0 .
\end{aligned}
$$

Now substitute the value of $\alpha$ as given by (20) into the preceding equation. The elimination leads to a polynomial of the seventh degree in $y$ which must be identically zero. Setting the coefficient of $y^{7}$ equal to zero, we find

$$
G^{2} G_{u}^{2} G_{u u}\left(G_{x x} G_{u u}-G_{x u}^{2}\right)=0 .
$$


We shall prove that $G_{u u}=0$. Assume the contrary. By (44), it then follows since $G_{u u} \neq 0$ that the surface $G=G(x, u)$ in the space with cartesian coordinates $(x, u, G)$ is developable. Hence $G$ is given parametrically by the equations

$$
\begin{aligned}
& G=f(\tau, K)+f_{\tau}(\tau, K)(u-\tau), \\
& x=g(\tau, K)+g_{\tau}(\tau, K)(u-\tau),
\end{aligned}
$$

where the subscript $\tau$ denotes partial differentiation with respect to $\tau$ only.

The second equation defines $\tau$ as a function of $x$ and $u$ only (provided that $g_{\tau \tau} \neq 0$ ). Its partial derivatives and also those of $G$ are

$$
\begin{aligned}
\tau_{x} & =1 / g_{\tau \tau}(u-\tau), & \tau_{u} & =-g_{\tau} / g_{\tau \tau}(u-\tau) ; \\
G_{x} & =f_{\tau \tau} / g_{\tau \tau}, & G_{u} & =f_{\tau}-g_{\tau} f_{\tau \tau} / g_{\tau \tau} .
\end{aligned}
$$

Next the function $\alpha$ as defined by (20) must satisfy the differential equation $\alpha_{K}=y \alpha_{u}$. Substituting this value of $\alpha$ into it, we discover a polynomial of the sixth degree in $y$ which must be identically zero. Upon setting the coefficient of $y^{6}$ equal to zero, we find

$$
\frac{\partial}{\partial u}\left[\frac{1}{G}\left(G_{x}-\frac{G_{u} G_{x u}}{G_{u u}}\right)\right]=0 .
$$

Substituting (45) and (46) into the preceding equation, we find that it becomes

$$
\frac{\partial}{\partial u}\left(\frac{f_{\tau}}{G g_{\tau}}\right)=0 .
$$

The expansion of this yields the fact that $f_{\tau} g_{\tau \tau}-f_{\tau \tau} g_{\tau}=0$. By (46) this means that $G_{u}=0$. This contradiction shows that in all cases $G_{u u}=0$.

Since $G_{u u}=0$, we find upon substituting the value of $\alpha$ as given by (20) into (43) a polynomial of the sixth degree in $y$ which must be identically zero. Upon setting the coefficient of $y^{6}$ equal to zero, we obtain

$$
3 G^{2} G_{u}^{2} G_{x u}^{2} G_{u K}=0 .
$$

We shall prove that $G_{x u}=0$. Suppose the contrary so that $G_{x u} \neq 0$. Then $G_{u K}=0$ and $G_{K K} \neq 0$. Therefore upon substituting the value of $\alpha$ as given by (20) into (43), we discover a polynomial of the fifth degree in $y$ which must be identically zero. Upon placing the coefficient of $y^{5}$ equal to zero, we find

$$
2 G G_{u}^{2} G_{x u}^{2}=0 .
$$

This is a contradiction of the fact that $G_{x u} \neq 0$. Hence in all cases $G_{u u}=G_{x u}=0$.

Under these conditions, we now find from (20) that $\alpha$ is given by the equation 


$$
\begin{aligned}
G\left(-2 y G_{u K}+G_{K K}\right) \alpha= & y^{2}\left(G_{u} G_{x K}-2 G_{x} G_{u K}-G_{u} H_{u}\right) \\
& +y\left(G_{x} G_{K K}-G_{K} G_{x K}+G_{K} H_{u}+G_{u} H_{K}-2 H G_{u K}\right) \\
& +\left(H G_{K K}-G_{K} H_{K}\right) .
\end{aligned}
$$

Substitute (51) into (43). We obtain a polynomial of the fifth degree in $y$. The leading coefficient must be zero so that

$$
4 G^{2} G_{u}^{2} G_{x x} G_{u K}^{2}=0 .
$$

10. Case 3. $G_{x x} \neq 0$. In this case, we find from the preceding equation that $G_{u K}=0$. Upon eliminating $\alpha$ from (51) and (43), we obtain a quadratic equation in $y$ which is identically zero. Upon placing the coefficient of $y^{2}$ equal to zero, we determine

$$
\left(G_{x K}-H_{u}\right)^{2}=G_{x x} G_{K K} .
$$

Next upon placing the value of $\alpha$ as given by (51) into the differential equation $\alpha_{K}-y \alpha_{u}=0$, we obtain a cubic equation in $y$ which is an identity. Upon setting the leading term equal to zero, we discover in conjunction with (53) and the other conditions that

$$
\frac{\partial}{\partial u}\left[\frac{G_{u}\left(G_{x K}-H_{u}\right)}{G G_{K K}}\right]^{2}=\frac{\partial}{\partial u}\left[\frac{G_{u}^{2} G_{x x}}{G^{2} G_{X K}}\right]=0 .
$$

Carrying out the differentiation, we discover that $G_{u}=0$. Therefore $G$ depends upon $x$ and $K$ only.

The equations (20) and (43) become in this case

$$
\begin{aligned}
G G_{K K} \alpha & =y\left[G_{x} G_{K K}-G_{K}\left(G_{x K}-H_{u}\right)\right]+\left(H G_{K K}-G_{K} H_{K}\right), \\
G\left(G_{x K}-H_{u}\right) \alpha & =y\left[G_{x}\left(G_{x K}-H_{u}\right)-G_{K} G_{x x}\right]+\left[H\left(G_{x K}-H_{u}\right)-G_{K} H_{x}\right],
\end{aligned}
$$

where, of course, $G=G(x, K)$ only, $G_{K K} \neq 0$, and $G_{x x} \neq 0$.

Eliminating $\alpha$ from these two equations, we obtain a linear equation in $y$ which must be identically zero. Also using the fact that $\alpha$ as defined by the first of the preceding equations satisfies the equation $\alpha_{K}=y \alpha_{u}$, we find that in totality $G$ and $H$ must satisfy the four partial differential equations

$$
\begin{gathered}
\left(G_{x K}-H_{u}\right)^{2}=G_{x x} G_{K K}, \quad H_{K}\left(G_{x K}-H_{u}\right)=H_{x} G_{K K}, \\
G_{x} G_{K K}-G_{K}\left(G_{x K}-H_{u}\right)=G G_{K K}(a K+b), \\
H G_{K K}-G_{K} H_{K}=G G_{K K}(a u+c),
\end{gathered}
$$

where $(a, b, c)$ are functions of $x$ only. We shall now consider this system of partial differential equations.

In the first place, it is noted that by solving the first of equations (56) for $H_{u}$, the expression $H_{u}$ is independent of $u$. Hence $H$ is linear integral in $u$ with coefficients functions of $(x, K)$ only. 
Integrating the last of equations (56) and using the fact that $H$ is linear integral in $u$, we see that $H$ is given by an expression of the form

$$
H=(a u+c)\left(G-K G_{K}\right)+G_{K}(d u+f),
$$

where $d$ and $f$ are functions of $x$ only.

Substituting this value of $H$ into the third of equations (56) and integrating the result with respect to $K$, we find that $G$ must satisfy the first order partial differential equation

$$
G_{x}=(a K+b)\left(G-K G_{K}\right)+G_{K}(d K+g),
$$

where $g$ is a function of $x$ only.

By equations (19), (55), (56), (57), and (58), we find that $\alpha$ and $\beta$ are given by

$$
\alpha=a z+b y+c, \quad \beta=d z+g y+f,
$$

where, of course, $(a, b, c, d, g, f)$ are functions of $x$ only.

Now by the last of the equations (13), we find that since $G_{K K} \neq 0$, the functions $\alpha$ and $\beta$ must satisfy the two first order partial differential equations

$$
\alpha_{x}+\alpha \alpha_{y}+\beta \alpha_{z}=0, \quad \beta_{x}+\alpha \beta_{y}+\beta \beta_{z}=0 .
$$

Substituting (59) into these equations, we obtain two linear equations in $y$ and $z$ which must be identically zero. Thus we obtain the equations

$$
\begin{array}{rrrl}
a_{x}+a(b+d)=0, & b_{x}+b^{2}+a g=0, & c_{x}+b c+a f=0, \\
d_{x}+d^{2}+a g=0, & g_{x}+g(b+d)=0, & f_{x}+c g+d f=0 .
\end{array}
$$

Next substituting (57) into the second of equations (56), we have upon equating the coefficients of $u$

$$
\begin{aligned}
a G_{x} & =\left[a(a K-d)-a_{x}\right]\left(G-K G_{K}\right)+G_{K}\left[d(a K-d)-d_{x}\right], \\
c G_{x} & =\left[a(c K-f)-c_{x}\right]\left(G-K G_{K}\right)+G_{K}\left[d(c K-f)-f_{x}\right] .
\end{aligned}
$$

Eliminating $G_{x}$ from (58) and (62), we find upon making use of (61) and the fact that $G_{K K} \neq 0$ that $a$ must be a constant.

Therefore by. (61) either $a$ or $(b+d)$ is zero. We shall divide these possibilities into various cases.

First let $a=a_{0} \neq 0$. Thence by (61), we have $d=-b=-b_{0}$ where $b_{0}$ is constant. From (61), we also have

$$
g=-b_{0}^{2} / a_{0}, \quad c=c_{0} x+c_{1}, \quad f=-\left(1 / a_{0}\right)\left[c_{0}+b_{0}\left(c_{0} x+c_{1}\right)\right],
$$

where $\left(a_{0}, b_{0}, c_{0}, c_{1}\right)$ are constants. Substituting these into (59), we find that $\alpha$ and $\beta$ are given by

$$
\alpha=a_{0} z+b_{0} y+c_{0} x+c_{1}, \quad \beta=-\left(1 / a_{0}\right)\left(b_{0} \alpha+c_{0}\right) .
$$


The lines of force are therefore the straight lines

$$
a_{0} z+b_{0} y+c_{0} x+c_{1}=\lambda_{0}, \quad y=\lambda_{0} x+\mu_{0},
$$

where $\left(\lambda_{0}, \mu_{0}\right)$ are the constants of integration. Since the straight lines are in the parallel pencil of planes given by the first of the preceding equations, this type comes under Type (I) of our Fundamental Theorem.

Next consider the case where $a=0$ but $b \neq 0$ and $d \neq 0$. By equations (61),

$$
\begin{aligned}
& b=1 /\left(x-x_{0}\right), \quad d=1 /\left(x-x_{1}\right), \quad c=-y_{0} /\left(x-x_{0}\right), \\
& g=g_{0} /\left(x-x_{0}\right)\left(x-x_{1}\right), \quad f=-y_{0} g_{0} /\left(x-x_{0}\right)\left(x-x_{1}\right)-z_{0} /\left(x-x_{1}\right),
\end{aligned}
$$

where $\left(x_{0}, x_{1}, y_{0}, g_{0}, z_{0}\right)$ are constants. Substituting these into (59), we discover that $\alpha$ and $\beta$ are given by

$$
\alpha=\frac{y-y_{0}}{x-x_{0}}, \quad \beta=\frac{z-z_{0}}{x-x_{1}}+\frac{g_{0}\left(y-y_{0}\right)}{\left(x-x_{0}\right)\left(x-x_{1}\right)} .
$$

The lines of force are again the straight lines

$$
y-y_{0}=\lambda_{0}\left(x-x_{0}\right), \quad z-z_{0}+g_{0} \lambda_{0}=\mu_{0}\left(x-x_{1}\right),
$$

where $\left(\lambda_{0}, \mu_{0}\right)$ are the constants of integration. Since the straight lines are in the pencil of planes given by the first of the preceding equations, this type comes under Type (I) of our Fundamental Theorem.

Let us now examine the case where $a=b=0$ but $d \neq 0$. By equations (61),

$$
d=\frac{1}{x-x_{0}}, \quad g=\frac{g_{0}}{x-x_{0}}, \quad c=c_{0}, \quad f=-\frac{c_{0} g_{0} x+z_{0}}{x-x_{0}} .
$$

From (59), we find $\alpha=c_{0}, \beta=\left[\left(z-z_{0}\right)+g_{0}\left(y-c_{0} x\right)\right] /\left(x-x_{0}\right)$. The lines of force are then the straight lines

$$
y=c_{0} x+\lambda_{0}, \quad z-z_{0}+g_{0} \lambda_{0}=\mu_{0}\left(x-x_{0}\right) .
$$

These are straight lines parallel to the plane $y=c_{0} x$ and intersecting the straight line $x=x_{0}, z=-g_{0} y+z_{0}+c_{0} g_{0} x_{0}$. These come under the Type (I) of our Fundamental Theorem.

Again let us examine the case where $a=d=0$ but $b \neq 0$. By equations (61),

$$
b=\frac{1}{x-x_{0}}, \quad g=\frac{g_{0}}{x-x_{0}}, \quad c=\frac{-y_{0}}{x-x_{0}}, \quad f=\frac{-y_{0} g_{0}}{x-x_{0}}+f_{0},
$$

where $\left(x_{0}, y_{0}, g_{0}, f_{0}\right)$ are constants. The functions $\alpha$ and $\beta$ are given by $\alpha=\left(y-y_{0}\right) /\left(x-x_{0}\right), \beta=f_{0}+g_{0} \alpha$. The lines of force are the straight lines

$$
y-y_{0}=\lambda_{0}\left(x-x_{0}\right), \quad z=\left(f_{0}+g_{0} \lambda_{0}\right) x+\mu_{0} .
$$

These straight lines are in the pencil of planes given by the first of the above equations. Thus we have again the Type (I) of our Fundamental Theorem. 
Finally let $a=b=d=0$. By (61), we find that $c$ and $g$ are constants. The functions $\alpha$ and $\beta$ are

$$
\alpha=c_{0}, \quad \beta=g_{0}\left(y-c_{0} x\right)+f_{0},
$$

where $\left(c_{0}, g_{0}, f_{0}\right)$ are constants. The lines of force are the straight lines

$$
y=c_{0} x+\lambda_{0}, \quad z=\left(g_{0} \lambda_{0}+f_{0}\right) x+\mu_{0} .
$$

These straight lines are in the parallel pencil of planes given by the first of the above equations. Thus these come under the Type (I) of our Fundamental Theorem.

Thus the full discussion of Case 3 demonstrates that we have but special cases of the Type (I) of our Fundamental Theorem.

11. All the remaining cases are such that

$$
G_{x x}=G_{x u}=G_{u u}=0 .
$$

Under these conditions, substitute the value of $\alpha$ as given by (51) into (43). The result is a polynomial of the fourth degree in $y$ which is an identity. The leading term is zero so that we find

$$
G^{2} G_{u}^{2} G_{u K}\left[\left(G_{x K}-H_{u}\right)^{2}+4 H_{x} G_{u K}\right]=0 .
$$

12. Case 4. $G_{u K} \neq 0$. Upon substituting (76) into (43), we find that (43) becomes a perfect square. Upon solving this perfect square for $\alpha$, we find

$$
2 G_{u K} \alpha=\left(G_{x K}-B_{u}\right)\left(G_{K}-y G_{u}\right)+2 G_{u K}\left(y G_{x}+H\right) .
$$

Upon eliminating $\alpha$ between (51) and (77), we obtain a quadratic equation in $y$ which must be an identity. Upon setting all the various coefficients equal to zero, we obtain the two partial differential equations in $G$ and $H$

$$
\begin{aligned}
\left(G_{x K}-H_{u}\right)^{2}+4 H_{x} G_{u K} & =0, \\
G_{K K}\left(G_{x K}-H_{u}\right)+2 H_{K} G_{u K} & =0 .
\end{aligned}
$$

Next the value of $\alpha$ as given by (77) must satisfy the equation $\alpha_{K}=y \alpha_{u}$. Therefore it follows that

$$
\begin{aligned}
-G_{u}\left(G_{x K}-H_{u}\right)+2 G_{x} G_{u K} & =2 G G_{u K}(a K+b), \\
G_{K}\left(G_{x K}-H_{u}\right)+2 H G_{u K} & =2 G G_{u K}(a u+c),
\end{aligned}
$$

where $(a, b, c)$ are functions of $x$ only. We can solve the preceding two equations for $\left(G_{x K}-H_{u}\right)$ and $H$ obtaining the relations

$$
\begin{aligned}
G_{x K}-H_{u} & =\frac{2 G_{x} G_{u K}}{G_{u}}-\frac{2 G G_{u K}}{G_{u}}(a K+b), \\
H & =G(a u+c)+\frac{G G_{K}}{G_{u}}(a K+b)-\frac{G_{x} G_{K}}{G_{u}} .
\end{aligned}
$$


Eliminating $H$ from these two equations, we find

$$
\begin{aligned}
G_{x K}-a G-G_{u}(a u+c)-G_{K}(a K+b) & \\
& +\frac{G G_{u K}}{G_{u}}(a K+b)-\frac{G_{x} G_{u K}}{G_{u}}=0 .
\end{aligned}
$$

Differentiating this partially with respect to $u$, we find $-2 a G_{u}=0$. Hence in all cases we have $a=0$.

Upon substituting $a=0$, we can solve (81) for $c$. Substituting this value of $c$ in (80), we obtain the following expressions

$$
\begin{aligned}
G_{u}^{2} c & =\left(G_{u} G_{x K}-G_{x} G_{u K}\right)+b\left(G G_{u K}-G_{u} G_{K}\right), \\
G_{u}^{2} H & =G\left(G_{u} G_{x K}-G_{x} G_{u K}\right)-G_{x} G_{u} G_{K}+b G^{2} G_{u K} .
\end{aligned}
$$

This value of $H$ will of course satisfy the second of equations (78). Upon substituting this value of $H$ into the first of equations (78), we find

$$
b^{2}+b_{x}=0 .
$$

Let us first of all consider the case where $b=0$. We know that in all cases $G$ is given by the expression

$$
G=x l(K)+u m(K)+n(K),
$$

where $(l, m, n)$ are functions of $K$ only. From the first of equations (82), we discover that $c(x)=\left(l_{K} m-l m_{K}\right) / m^{2}$. Therefore $c=c_{0}$ and $l=\left(c_{0} K+c_{1}\right)$. The functions $G$ and $H$ are then given by

$$
\begin{aligned}
G & =m\left[\left(c_{0} K+c_{1}\right) x+u\right]+n, \\
H & =c_{0} G-\left(c_{0} K+c_{1}\right) G_{K} .
\end{aligned}
$$

Substituting these into the equation (77) defining $\alpha$ and in to the equation (19) defining $\beta$, we discover that $\alpha=c_{0}$ and $\beta=c_{1}$. Thus the field of force is of the parallel type as given by Type (III) in our Fundamental Theorem.

Next consider the case where $b \neq 0$. From (83), we find that $b=1 /\left(x-x_{0}\right)$, where $x_{0}$ is a constant. By the first of equations (82), we find upon using (84) that

$$
c(x)=-\frac{x_{0} \partial(l / m) / \partial K+\partial(n / m) / \partial K}{\left(x-x_{0}\right)} .
$$

From this, we deduce that

$$
\begin{aligned}
c & =-y_{0} /\left(x-x_{0}\right), \quad n=-x_{0} l+\left(y_{0} K-z_{0}\right) m, \\
G & =l\left(x-x_{0}\right)+m\left(u+y_{0} K-z_{0}\right), \\
m^{2} H & =G\left(m l_{K}-\operatorname{lm} m_{K}\right)-\operatorname{lm} G_{K}+m_{K} G^{2} /\left(x-x_{0}\right) .
\end{aligned}
$$


Substituting these into the equation (77) defining $\alpha$ and into the equation (19) defining $\beta$, we find

$$
\alpha=\left(y-y_{0}\right) /\left(x-x_{0}\right), \quad \beta=\left(z-z_{0}\right) /\left(x-x_{0}\right) .
$$

Thus in this case we have the general central fields of force yielding the Type (III) of our Fundamental Theorem.

13. Case 5. $G_{u K}=0$. In this case the equation (51) defining $\alpha$ now assumes the form

$$
\begin{aligned}
G G_{K K} \alpha= & y^{2} G_{u}\left(G_{x K}-H_{u}\right)+y\left[G_{x} G_{K K}+G_{u} H_{K}-G_{K}\left(G_{x K}-H_{u}\right)\right] \\
& +\left(H G_{K K}-G_{K} H_{K}\right) .
\end{aligned}
$$

The equation (43) becomes

$$
G\left(G_{x K}-H_{u}\right) \alpha=-H_{x}\left(G_{K}-y G_{u}\right)+\left(y G_{x}+H\right)\left(G_{x K}-H_{u}\right) .
$$

Assume $G_{u} \neq 0$. Upon eliminating $\alpha$ from the preceding two equations, we obtain a quadratic equation in $y$ which is an identity. Upon setting the various coefficients equal to zero, we find

$$
G_{x K}-H_{u}=0, \quad H_{x}=0 .
$$

If $G_{u}=0$, we discover upon eliminating $\alpha$ a linear equation in $y$ which must be identically zero. Upon setting the coefficients equal to zero, we find that the equations (91) are valid whether $G_{u} \neq 0$ or $G_{u}=0$.

By (91), it follows that $H$ is given by

$$
H=u l_{K}+f(K) .
$$

By (84) and (89), we find that, since $\alpha$ must satisfy the equation $\alpha_{K}=y \alpha_{u}$, $G$ and $H$ must satisfy the two equations

$$
\begin{aligned}
l G_{K K}+m H_{K} & =G G_{K K}(a K+b), \\
H G_{K K}-G_{K} H_{K} & =G G_{K K}(a u+c),
\end{aligned}
$$

where $(a, b, c)$ are functions of $x$ only.

Observe that $G_{u K}=m_{K}=0$. Hence $m=m_{0}$, a constant. Substituting (84) and (92) into the preceding equations, we find

$$
\begin{aligned}
l\left(l_{K K} x+n_{K K}\right)+m_{0}\left(u l_{K K}+f_{K}\right) & =\left(l x+m_{0} u+n\right)\left(l_{K K} x+n_{K K}\right)(a K+b), \\
\left(u l_{K}+f\right)\left(l_{K K} x+n_{K K}\right)-\left(u l_{K K}+f_{K}\right)\left(l_{K} x+n_{K}\right) & =\left(l x+m_{0} u+n\right)\left(l_{K K} x+n_{K K}\right)(a u+c) .
\end{aligned}
$$

The preceding equations must be identities in $u$. Upon setting the coefficient of $u^{2}$ in the last of the above equations equal to zero, we find $a m_{0}\left(l_{K K} x+n_{K K}\right)=0$. Since $G_{K K}=l_{K K} x+n_{K K} \neq 0$, we discover that $a m_{0}=0$.

We shall prove that $a=0$. For suppose this is not so. Since $a \neq 0$, it follows by the preceding paragraph that $m_{0}=0$. Upon setting the coefficient of the 
first degree term in $u$ of the last of the equations (94) equal to zero, we find

$$
l_{K} n_{K K}-l_{K K} n_{K}=a(l x+n)\left(l_{K K} x+n_{K K}\right) \text {. }
$$

If $l_{K K} \neq 0$ so that $l \neq 0$, it follows from this that the ratio $n / l$ is constant (since $a$ must be a function of $x$ only). But this will make the left-hand side of the preceding equation zero and hence $a=0$. This contradiction shows that $l_{K K}=0$.

Since $l_{K K}=0$, it follows that $n_{K K} \neq 0$ since $G_{K K}=l_{K K} x+n_{K K} \neq 0$. The equation (95) may then be written as

$$
l_{K}=a(l x+n) .
$$

Now if $l_{K} \neq 0$, we see from this equation that $l_{K} / l$ must be constant. Hence since $l_{K K}=0$ and $l_{K} / l$ is constant, it follows that $l_{K}=0$. It therefore follows that $a=0$. This is impossible so that in all cases we have proved that $a=0$.

Since $a=0$, we find that the equations (94) are equivalent to

$$
\begin{aligned}
l_{K K} & =\left(l_{K K} x+n_{K K}\right) b, \\
l\left(l_{K K} x+n_{K K}\right)+m_{0} f_{K} & =(l x+n)\left(l_{K K} x+n_{K K}\right) b, \\
l_{K} n_{K K}-l_{K K} n_{K} & =m_{0}\left(l_{K K} x+n_{K K}\right) c, \\
f\left(l_{K K} x+n_{K K}\right)-f_{K}\left(l_{K} x+n_{K}\right) & =(l x+n)\left(l_{K K} x+n_{K K}\right) c .
\end{aligned}
$$

First let us consider the case where $m_{0}=0$ and $l=0$ so that $n_{K K} \neq 0$. From the preceding equations, we find

$$
b=0, \quad n n_{K K} c=f n_{K K}-f_{K} n_{K} .
$$

Thus the quantity $c$ must be a constant $c_{0}$. Integrating the above differential equation, we find

$$
f=c_{0} n-c_{0} K n_{K}+c_{1} n_{K},
$$

where $c_{0}$ and $c_{1}$ are constants. By (89), (92), (93), and (19), we find that $\alpha=c_{0}$ and $\beta=c_{1}$. Therefore this case yields a parallel field of force and belongs to the Type (III).

Next let us consider the case where $m_{0}=0$ and $l \neq 0$. From the first three of the equations (97), we find

$$
n l_{K K}-\ln _{K K}=0, \quad n_{K} l_{K K}-l_{K} n_{K K}=0 .
$$

Since $l_{K K}$ and $n_{K K}$ are both not zero, it follows from these equations that $l n_{K}-n l_{K}=0$. Since $l \neq 0$, we find that $n=-x_{0} l$ where $x_{0}$ is a constant. The equations (97) may be written as

$$
b=\frac{1}{x-x_{0}}, \quad c=\frac{l_{K K} f-l_{K} f_{K}}{l l_{K K}\left(x-x_{0}\right)} .
$$

Of course $l_{K K} \neq 0$ since $G_{K K}=l_{K K}\left(x-x_{0}\right) \neq 0$. Since $c$ is a function of $x$ only, we then find 


$$
l_{K K} f-l_{K} f_{K}=-y_{0} l_{K K},
$$

where $y_{0}$ is a constant. The in tegration of this yields

$$
f=-y_{0} l+l_{K}\left(y_{0} K-z_{0}\right) \text {. }
$$

By (89), (92), and (19), we find

$$
\alpha=\left(y-y_{0}\right) /\left(x-x_{0}\right), \quad \beta=\left(z-z_{0}\right) /\left(x-x_{0}\right) .
$$

Thus we obtain the central fields of force and these belong to the Type (III).

Finally we discuss the case where $m_{0} \neq 0$. The equations (97) are then equivalent to

$$
\begin{aligned}
l_{K K} & =\left(l_{K K} x+n_{K K}\right) b, \\
l_{K} n_{K K}-l_{K K} n_{K} & =m_{0}\left(l_{K K} x+n_{K K}\right) c, \\
m_{0} f & =n l_{K}-\ln _{K} .
\end{aligned}
$$

If $l_{K K}=0$ so that $l=l_{0} K+l_{1}$, then $n_{K K} \neq 0$. Hence $b=0, c=l_{0} / m_{0}$, $G=\left(l_{0} K+l_{1}\right) x+m_{0} u+n(K)$, and $H=l_{0} u+\left(1 / m_{0}\right)\left[n l_{0}-n_{K}\left(l_{0} K+l_{1}\right)\right]$.

The values of $\alpha$ and $\beta$ are then

$$
\alpha=l_{0} / m_{0}, \quad \beta=-l_{1} / m_{0} .
$$

Thus we have the parallel fields of force of the Type (III).

Finally let $l_{K K} \neq 0$. By the first of equations (105), we see that $n_{K K} / l_{K K}$ is constant. The integration of this yields

$$
n=-x_{0} l+m_{0} y_{0} K-m_{0} z_{0},
$$

where $\left(x_{0}, y_{0}, z_{0}\right)$ are constants. The equations (105) are then equivalent to

$$
\begin{gathered}
b=1 /\left(x-x_{0}\right), \quad c=-y_{0} /\left(x-x_{0}\right), \\
f=l_{K}\left(y_{0} K-z_{0}\right)-y_{0} l .
\end{gathered}
$$

By (89), (92), (93), and (19), we find

$$
\alpha=\left(y-y_{0}\right) /\left(x-x_{0}\right), \quad \beta=\left(z-z_{0}\right) /\left(x-x_{0}\right) .
$$

Thus the final case belongs to the Type (III).

14. Conclusion. Our Fundamental Theorem has been completely proved. In conclusion, it is noted that the three distinct types of our theorem may be characterized in the following way. They are those families of dynamical trajectories whose $\infty^{5}$ curves can be analyzed into a series of sets, each set containing $\infty^{4}$ curves, in such a way that one of the sets will generate the others by the simple process of multiplication of curvatures described above.

IlLiNois Institute of TeChNoLogy,

Chicago, Ill. 